\title{
Energy principle and nonlinear electric-mechanical behavior of ferroelectric ceramics
}

\author{
F. Liu, H. J. Li, T. C. Wang \\ LNM, Institute of Mechanics, Chinese Academy of Sciences, Beijing, P.R. China \\ Received 12 June 2007; Accepted 15 October 2007; Published online 14 February 2008 \\ (C) Springer-Verlag 2008
}

\begin{abstract}
Summary. Many experimental observations have shown that a single domain in a ferroelectric material switches by progressive movement of domain walls, driven by a combination of electric field and stress. The mechanism of the domain switch involves the following steps: initially, the domain has a uniform spontaneous polarization; new domains with the reverse polarization direction nucleate, mainly at the surface, and grow though the crystal thickness; the new domain expands sideways as a new domain continues to form; finally, the domain switch coalesces to complete the polarization reversal. According to this mechanism, the volume fraction of the domain switching is introduced in the constitutive law of the ferroelectric material and used to study the nonlinear constitutive behavior of a ferroelectric body in this paper. The principle of stationary total potential energy is put forward in which the basic unknown quantities are the displacement $u_{i}$, electric displacement $D_{i}$ and volume fraction $\rho_{I}$ of the domain switching for the variant $I$. The mechanical field equation and a new domain switching criterion are obtained from the principle of stationary total potential energy. The domain switching criterion proposed in this paper is an expansion and development of the energy criterion established by Hwang et al. [1]. Based on the domain switching criterion, a set of linear algebraic equations for determining the volume fraction $\rho_{I}$ of domain switching is obtained, in which the coefficients of the linear algebraic equations only contain the unknown strain and electric fields. If the volume fraction $\rho_{I}$ of domain switching for each domain is prescribed, the unknown displacement and electric potential can be obtained based on the conventional finite element procedure. It is assumed that a domain switches if the reduction in potential energy exceeds a critical energy barrier. According to the experimental results, the energy barrier will strengthen when the volume fraction of the domain switching increases. The external mechanical and electric loads are increased step by step. The volume fraction $\rho_{I}$ of domain switching for each element obtained from the last loading step is used as input to the constitutive equations. Then the strain and electric fields are calculated based on the conventional finite element procedure. The finite element analysis is carried out on the specimens subjected to uniaxial coupling stress and electric field. Numerical results and available experimental data are compared and discussed. The present theoretic prediction agrees reasonably with the experimental results.
\end{abstract}

\section{Introduction}

Ferroelectric materials are used in many electric components such as sensors, actuators and transducers owing to their coupled electromechanical character. Recent applications of ferroelectric

Correspondence: T. C. Wang, LNM, Institute of Mechanics, Chinese Academy of Sciences, Beijing 100080, P.R. China

e-mail: tcwang@imech.ac.cn 
materials include nonvolatile computer memories and switching capacitors for integrated circuitry. Those applications require the largest possible strain and polarization, and many repeated cycles of domain switch under high electric fields and high stress. Therefore, being able to understand and predict the change of materials response under combined electrical and mechanical loading is crucial for the suitable design of a ferroelectric device.

Significant effort has been devoted to the development of constitutive models to describe the nonlinear response of ferroelectric materials. Many theories were proposed to explain the nonlinear behavior of the ferroelectric ceramics. Those theories of the ferroelectric ceramics may be divided broadly into two types. Micromechanics and phenomenological modeling are the most common approaches to the development of constitutive models.

Micromechanics models are based on the microscopic behavior of the domains. Domain switching is considered the source of the butterfly shaped strain versus electric field curves and the corresponding electric displacement versus electric field loops. If the electrical and mechanical energy reduction of each domain exceeds a critical energy barrier, then switching occurs. The macroscopic behavior is controlled by the combination of the average microscopic switching behavior and the reversible linear behavior [1]-[24]. Early a micro-electro-mechanical model was proposed by Hwang et al. [1]. They established the energy criterion of the domain switching using the energy difference before and after the domain switching,

$E_{k} \Delta D_{k}^{s}+\sigma_{i j} \Delta \gamma_{i j}^{s} \geq 2 E_{c} P_{s}$

where $\Delta \gamma_{i j}^{s}$ and $\Delta D_{k}^{s}$ are the changes of spontaneous strain and spontaneous electric displacement before and after the domain switching, respectively, $E_{c}$ is the coercive electric field, $P_{s}$ is the spontaneous polarization. Lu et al. [2] developed Hwang's switching criterion [1]. They distinguished the energy barriers of $90^{\circ}$ and $180^{\circ}$ domain switching. The results given by them were matched well with those under uniaxial mechanical loading. Chen et al. [3] introduced the volume fraction of various kinds of domains as internal variables describing the pattern of the internal rearrangement resulting from the domain switching and studied the nonlinear behavior of polycrystalline ferroelectrics. Huo and Jiang [4] considered that every crystal had different kinds of domains and that the volume fractions of the various domains were the internal variables, so the average polarization of each crystal could be expressed as a linear function of the volume fraction, and the domain switching corresponds to changes of the volume fraction of domains. Huber et al. [5] developed the ferroelectric constitutive model based on the domain wall motion that is similar to the crystal slip. They thought that there were several variants in a tetragonal crystal and the volume fraction of each variant was $c_{I}$. They used a self-consistent analysis to estimate the macroscopic response of tetragonal crystals under a variety of loading paths. Huber and Fleck [6], [7] improved this model. Li and Weng [8]-[11] adopted the irreversible thermodynamics and physics of domain switch to study the nonlinear behavior of PZT, and it was recently expanded to include the effect of temperature to study the shift of Curie point (Su and Weng [12], [13]) and to calculate the hysteresis behavior of single crystals and the ceramic polycrystal (Srivastava and Weng [14], [15]; Su and Weng [16]). The micro-electro-mechanical models described some aspects of the behavior of ferroelectric materials [17]-[24]. The micro-electro-mechanical model reflects the physical essence of the nonlinear behavior of the ferroelectric material. But in order to make the calculation accurate, those methods require thousands of crystallite grains. Hence the finite element simulations cost too much computation time and are not efficient. A detailed review on recent advances in the micromechanical modeling of ferroelectric material was given by Huber [25].

Phenomenological theories start with a chosen set of state variables and an expression which connects them, such as a free-energy function. Thermodynamic principles are then used to derive 
further expressions. Chen and his cooperators [26]-[29] proposed the phenomenological model to describe the electromechanical coupling characters of ferroelectric material. Their model was quite complex and limited to uniaxial electrical loading. However, it did capture the fundamental butterfly and D-E loops and was capable of capturing some rate dependent behavior. A later model presented a 3D formulation, but gave no specific equations. Another macroscopic model, developed by Bassiouny et al. [30], [31], was based on the principle of virtual power from rational thermodynamics. They established the admissibility of additive decomposition of polarization and strain, as well as the use of remanent strain and polarization as internal state variables. The Clausius-Duhem inequality is then used as the basis for the development of the evolution of these internal variables by means of a dissipation potential. A simple macroscopic constitutive model with remanent polarization and remanent strain as internal variables was developed by Kamlah and his coworkers [32]-[35]. A series of work on constitutive modeling of ferroelectric ceramics has been published recently by McMeeking's group [36]-[41]. They established some selfconsistent analysis to estimate the macroscopic response of tetragonal crystals under a variety of loading paths. There have also been assorted other approaches to capturing the nonlinear behavior of these materials. Some other studies have also been conducted on ferroelectric constitutive relations [42]-[45].

Recent phenomenological models have had the aim of capturing the multi-axial behavior of ferroelectric nonlinear constitutive laws, with varying degrees of success. For instance, Kamlah and Tsakmakis [32] apply a bilinear approach, but because of the lack of multi-axial data, the model was only fully developed for the uniaxial case. In the plasticity based model by McMeeking and Landis [38], only three internal variables are employed by linking the remanent strain to the remanent polarization. Landis [39] proposed a multi-axial constitutive model. In the model an appropriate switching surface and associated flow rules for increments of remnant strains and polarization are proposed to make the model thermodynamically consistent. The reader is referred to the contribution by Kamlah [46] and Landis [47] for a survey of the thermodynamically consistent modeling of switching effects.

Early finite element models for piezoelectric materials utilized linear constitutive laws, and were only suitable for small stress and electric field loadings. At this time there have been lots of research activities in developing nonlinear finite element simulation for ferroelectric materials. Gong and Suo [48] and Hom and Shankar [49] simulated the electrostriction behavior of multilayer actuators. When analyzing the interaction between adjacent domains, a straightforward approach is to employ a micromechanical model for single domain behavior at each integration point in a finite element scheme. The finite element mesh can then be used to capture the details of grain geometry, with the orientation of the direction of polarization varying from domain to domain. Based on this idea, the interaction among grains has been implemented in the finite element method for simulating the fully coupled constitutive behavior by Hwang et al. [19]; Lu et al. [2]; Hwang and Waser [50]; Fang et al. [51]. Liu Bin [52] analyzed the plane problem using total coupling nonlinear finite element method. Kamlah and Bohle [33] implemented a phenomenological law into a finite element code. Their finite element tool is suitable for studying the influence of geometry and material parameters on the stresses in critical regions of piezoceramic devices. Ghandi and Hagood [53] developed a hybrid finite element formulation, which incorporates electric displacement degrees of freedom along with the conventional displacement and electric potential degrees of freedom. Landis [54] developed a new finite element formulation, the vector potential formulation which uses a charge based potential as the independent variable. Li and Fang [55] carried out 3D finite element simulations on ferroelectric materials. Kim and Jiang [56] and Arockiarajan et al. [57] also developed a 3D finite element model for rate-dependent behavior of ferroelectric ceramics. 


\section{Constitutive law and energy principle}

\subsection{The volume fraction of domain switching}

First consider the domain switches only in one direction. Suppose the material element is a single domain zone before switching. The spontaneous polarization, spontaneous polarization strain, elastic compliance, dielectric and piezoelectricity coefficients are $D_{i}^{s(0)}, \gamma_{i j}^{s(0)}, S_{i j m n}^{(0)}, \varepsilon_{i j}^{(0)}$ and $d_{i j k}^{(0)}$, respectively. The corresponding quantities will be $D_{i}^{s(I)}, \gamma_{i j}^{s(I)}, S_{i j m n}^{(I)}, \varepsilon_{i j}^{(I)}$ and $d_{i j k}^{(I)}$ after the whole domain in the material element has switched. If the volume friction of the switched domain in the material element is $\rho_{I}$, the spontaneous polarization, spontaneous strain and the piezoelectric, elastic and dielectric moduli of the material element will be

$D_{i}^{S}=\left(1-\rho_{I}\right) D_{i}^{s(0)}+\rho_{I} D_{i}^{s(I)}=D_{i}^{s(0)}+\rho_{I} \Delta D_{i}^{s(I)}$,

$\gamma_{i j}^{s}=\left(1-\rho_{I}\right) \gamma_{i j}^{s(0)}+\rho_{I} \gamma_{i j}^{s(I)}=\gamma_{i j}^{s(0)}+\rho_{I} \Delta \gamma_{i j}^{s(I)}$,

$d_{i k l}=\left(1-\rho_{I}\right) d_{i k l}^{(0)}+\rho_{I} d_{i k l}^{(I)}=d_{i k l}^{(0)}+\rho_{I} \Delta d_{i k l}^{(I)}$,

$S_{i j m n}=\left(1-\rho_{I}\right) S_{i j m n}^{(0)}+\rho_{I} \Delta S_{i j m n}^{(I)}=S_{i j m n}^{(0)}+\rho_{I} \Delta S_{i j m n}^{(I)}$,

$\varepsilon_{i j}=\left(1-\rho_{I}\right) \varepsilon_{i j}^{(0)}+\rho_{I} \Delta \varepsilon_{i j}^{(I)}=\varepsilon_{i j}^{(0)}+\rho_{I} \Delta \varepsilon_{i j}^{(I)}$,

where symbol $\Delta$ is the change of the related physical quantity before switching and after the whole domain of the material element having switched.

In fact, the domain may switch both in the $90^{\circ}$ and $180^{\circ}$ directions. And the domain may switch in several potential orientations during $90^{\circ}$ switching. Suppose the volume fraction of $180^{\circ}$ switching is $\rho_{1}$. If there are $N-190^{\circ}$ switchings, the volume fraction of $90^{\circ}$ switching can be expressed as $\rho_{I}$ $(I \in 2,3, \ldots, N)$.

Similar to Eq. (2), the spontaneous electric displacement the spontaneous strain and the piezoelectric, elastic and dielectric moduli of the material element can be written as follows:

$D_{i}^{s}=D_{i}^{s(0)}+\sum_{I=1}^{N} \rho_{I} \Delta D_{i}^{s(I)}$,

$\gamma_{i j}^{s}=\gamma_{i j}^{s(0)}+\sum_{I=1}^{N} \rho_{I} \Delta \gamma_{i j}^{s(I)}$,

$d_{i k l}=d_{i k l}^{(0)}+\sum_{I=1}^{N} \rho_{I} \Delta d_{i k l}^{(I)}$,

$S_{i j m n}=S_{i j m n}^{(0)}+\sum_{I=1}^{N} \rho_{I} \Delta S_{i j m n}^{(I)}$,

$\varepsilon_{i j}=\varepsilon_{i j}^{(0)}+\sum_{I=1}^{N} \rho_{I} \Delta \varepsilon_{i j}^{(I)}$,

where $I=1$ corresponds to $180^{\circ}$ switching, $I \in 2,3, \ldots, N$ are corresponding $90^{\circ}$ switchings in the $I$-th orientation. 
The constitutive law of the ferroelectric material can be described as follows:

$$
\begin{aligned}
& \gamma_{i j}-\gamma_{i j}^{s(0)}-\sum_{I=1}^{N} \rho_{I} \Delta \gamma_{i j}^{s(I)}=\left(S_{i j m n}^{(0)}+\sum_{I=1}^{N} \rho_{I} \Delta S_{i j m n}^{(I)}\right) \sigma_{m n}+\left(d_{k i j}^{(0)}+\sum_{I=1}^{N} \rho_{I} \Delta d_{k i j}^{(I)}\right) E_{k}, \\
& D_{i}-D_{i}^{s(0)}-\sum_{I=1}^{N} \rho_{I} \Delta D_{i}^{s(I)}=\left(d_{i k l}^{(0)}+\sum_{I=1}^{N} \rho_{I} \Delta d_{i k l}^{(I)}\right) \sigma_{k l}+\left(\varepsilon_{i k}^{(0)}+\sum_{I=1}^{N} \rho_{I} \Delta \varepsilon_{i k}^{(I)}\right) E_{k},
\end{aligned}
$$

where $S_{i j k l}$ and $\varepsilon_{i k}$ are the elastic compliance and dielectric permittivity tensor measured at constant electric field and constant stress, respectively. Tensor $d_{i k l}$ is the piezoelectric coefficient at constant stress. In general the displacement $u_{i}$ and electric potential $\phi$ are used as the basic unknown quantities in finite element simulation. Hence the second type constitutive law is needed in which the basic unknown quantities are the strain $\gamma_{i j}$ and electric field $E_{i}$. We have

$\sigma_{i j}=C_{i j m n}\left(\gamma_{m n}-\gamma_{m n}^{s}\right)-C_{i j m n} d_{k m n} E_{k}$,

$D_{i}=D_{i}^{s}+C_{m n k l} d_{i k l}\left(\gamma_{m n}-\gamma_{m n}^{s}\right)+\left(\varepsilon_{i k}-d_{k m n} e_{i m n}\right) E_{k}$,

where $C_{i j m n}$ is the elastic modulus tensor measured at constant electric field, $e_{i m n}$ is the piezoelectric coefficient tensor at constant strain $e_{i m n}=C_{m n k l} d_{i k l}$.

\subsection{Principle of stationary total potential energy}

Suppose the volume and boundary of the ferroelectric body are $V$ and $S$, respectively. When no body forces and free charges are present, the field equations can be written as

$\sigma_{i j, j}=0$,

$D_{i, i}=0$.

The mechanical boundary conditions are:

$\sigma_{i j} n_{j}=\bar{t}_{i} \quad$ on $S_{\sigma}$,

$u_{i}=\bar{u}_{i} \quad$ on $S_{u}$,

where $\sigma_{i j}$ are the components of stress, $\bar{t}_{i}$ is the prescribed traction on $S_{\sigma}, \bar{u}_{i}$ is the prescribed displacement on $S_{u}$.

The electrical boundary conditions are:

$D_{i} n_{i}=-\bar{\omega} \quad$ on $S_{\omega}$,

$\phi=\bar{\phi} \quad$ on $S_{\phi}$,

where $D_{i}$ is the electric displacement, $\bar{\omega}$ is the prescribed surface free charge on $S_{\omega}, n_{i}$ is the unit normal to the surface $S$, and $\bar{\phi}$ is the prescribed electric potential on $S_{\phi}$.

The internal energy density can be expressed as follows [58]:

$U=\frac{1}{2}\left[\sigma_{i j}\left(\gamma_{i j}-\gamma_{i j}^{s(0)}-\sum_{I=1}^{N} \rho_{I} \Delta \gamma_{i j}^{s(I)}\right)+E_{k}\left(D_{k}-D_{k}^{s(0)}-\sum_{I=1}^{N} \rho_{I} \Delta D_{k}^{s(I)}\right)\right]$.

When the domain switching is considered, the total potential energy is

$\Phi=\int_{V} U d V-\int_{S_{\sigma}} \bar{t}_{\imath} u_{i} d S+\int_{S_{\phi}} \bar{\phi} D_{i} n_{i} d S+\int_{V}\left(\sum_{I=1}^{N} \rho_{I} W_{\mathrm{cr}}^{(I)}\right) d V$,

where $W_{\mathrm{cr}}^{(1)}$ is the critical energy barrier which the single domain per unit volume must overcome when the polarization of the whole domain has switched by $180^{\circ} . W_{\mathrm{cr}}^{(I)}(I=2,3, \ldots, N)$ is the critical 
energy barrier which the single domain per unit volume must overcome when the whole domain has switched by $90^{\circ}$ in the $I$-th variant orientation.

The first variation of the internal energy density is

$$
\begin{aligned}
\delta U= & \sigma_{i j} \delta \gamma_{i j}+E_{k} \delta D_{k} \\
& -\sum_{\mathrm{I}=1}^{N} \delta \rho_{\mathrm{I}}\left(\sigma_{i j} \Delta \gamma_{i j}^{s(I)}+E_{k} \Delta D_{k}^{s(I)}+\frac{1}{2} \Delta S_{i j m n}^{(I)} \sigma_{m n} \sigma_{i j}+\frac{1}{2} \Delta \varepsilon_{i k}^{(I)} E_{k} E_{i}+\Delta d_{k i j}^{(I)} \sigma_{i j} E_{k}\right) .
\end{aligned}
$$

The first variation of the total energy of the system is

$$
\delta \Phi=\int_{V} \delta U d V-\int_{S_{\sigma}} \bar{t}_{i} \delta u_{i} d S+\int_{S_{\phi}} \bar{\phi} \delta D_{i} n_{i} d S+\int_{V}\left(\sum_{I=1}^{N} \delta \rho_{\mathrm{I}} W_{\mathrm{cr}}^{(I)}\right) d V .
$$

Substituting Eq. (14) into Eq. (15), we get

$$
\begin{aligned}
\delta \Phi= & \int_{S_{\phi}} \bar{\phi} \delta D_{i} n_{i} d S-\int_{S_{\sigma}} \bar{t}_{i} \delta u_{i} d S+\int_{V}\left[\sigma_{i j} \delta \gamma_{i j}+E_{k} \delta D_{k}\right. \\
& \left.-\sum_{I=1}^{N} \delta \rho_{I}\left(\sigma_{i j} \Delta \gamma_{i j}^{S(I)}+E_{k} \Delta D_{k}^{s(I)}+\frac{1}{2} \Delta S_{i j m n}^{(I)} \sigma_{m n} \sigma_{i j}+\frac{1}{2} \Delta \varepsilon_{i k}^{(I)} E_{k} E_{i}+\Delta d_{k i j}^{(I)} \sigma_{i j} E_{k}-W_{\mathrm{cr}}^{(I)}\right)\right] d V .
\end{aligned}
$$

The displacement $u_{i}$ which satisfies the boundary condition (9) is called the kinematically admissible displacement, and the electric displacement $D_{i}$ which satisfies the field equation (7) and the boundary condition (10) is called the electrically admissible electric displacement. The volume fraction $\rho_{I}$ of the domain switching that satisfies the conditions $\rho_{I} \geq 0$ and $\sum_{I=1}^{N} \rho_{I} \leq 1$ is called the physically admissible volume fraction.

We have the following principle of stationary total energy:

Among all kinematically admissible displacements $u_{i}$, electrically admissible electric displacements $D_{i}$ and physically admissible volume fractions $\rho_{I}$, the actual displacement $u_{i}^{*}$, actual electric displacement $D_{i}^{*}$ and actual volume fraction $\rho_{I}^{*}$ make the total energy $\Phi$ stationary. The proof of the principle of stationary total energy is given in Appendix A.

Based on this principle of stationary total potential energy, one can obtain the stress field equation (6) and boundary conditions (8) and (11). In addition one can also obtain the following switching criterion:

$\sigma_{i j} \Delta \gamma_{i j}^{s(I)}+E_{k} \Delta D_{k}^{s(I)}+\frac{1}{2} \Delta S_{i j m n}^{(I)} \sigma_{m n} \sigma_{i j}+\frac{1}{2} \Delta \varepsilon_{i k}^{(I)} E_{k} E_{i}+\Delta d_{k i j}^{(I)} \sigma_{i j} E_{k}=W_{\mathrm{cr}}^{(I)}(I \in 1,2,3, \ldots)$.

It should be emphasized that Eq. (17) is only available for the variant $I$ in which the domain switching is active (It means that the volume fraction $\rho_{I}$ satisfies the active condition $\rho_{I}>0$ ). Equation (17) is the modification and further development of the energy criterion proposed by Hwang et al. [1]. It accords with the work by Kessler and Balke [58] and Huber and Fleck [6] in the spirit.

Since the most important contribution of material anisotropy in Eq. (17) is due to the term of $\Delta d_{k i j}^{(I)}$ $\sigma_{i j} E_{k}$, hence in order to simplify the finite element calculation procedure, following Hwang et al. [10], McMeeking and Landis [29], Kamlah [37] and Arockiarajan et al. [57], the ferroelectric material is assumed to be isotropic elastic and isotropic dielectric, but the piezoelectricity coefficient $d_{i j k}^{(I)}$ is anisotropic. 
Then Eq. (17) can be reduced to

$\sigma_{i j} \Delta \gamma_{i j}^{s(I)}+E_{k} \Delta D_{k}^{s(I)}+\Delta d_{k i j}^{(I)} \sigma_{i j} E_{k}=W_{\mathrm{cr}}^{(I)}$.

Substitute Eq. (5) into Eq. (18). A set of linear algebraic equations for $\rho_{I}$ are obtained,

$\sum_{J=1}^{N} \rho_{J} A_{I J}=\hat{W}_{\mathrm{cr}}^{(I)} \quad(I \in 1,2,3, \ldots, N)$,

where

$A_{I J}=C_{i j k l} \Delta \hat{\gamma}_{i j}^{(J)} \Delta \hat{\gamma}_{k l}^{(I)}$,

$\Delta \hat{\gamma}_{i j}^{(I)}=\Delta \gamma_{i j}^{s(I)}+\Delta d_{k i j}^{(I)} E_{k} \quad(I \in 1,2,3, \ldots, N)$,

$\hat{W}_{\mathrm{cr}}^{(I)}=\hat{\sigma}_{i j} \Delta \hat{\gamma}_{i j}^{(I)}+E_{k} \Delta D_{k}^{s(I)}-W_{\mathrm{cr}}^{(I)} \quad(I \in 1,2,3, \ldots, N)$,

$\hat{\sigma}_{m n}=C_{m n i j}\left(\gamma_{i j}-\gamma_{i j}^{s(0)}-d_{k i j}^{(0)} E_{k}\right)$.

It must be emphasized that the unknown quantities in the coefficients $A_{I J}$ are the strain and the electric field. Once the strain and electric field are given, the volume fraction $\rho_{I}$ of the domain switching can be determined by Eq. (19). Especially for a plane problem, $\rho_{1}$ is the volume fraction of $180^{\circ}$ switching, $\rho_{2}$ is the volume fraction of clockwise $90^{\circ}$ switching, $\rho_{3}$ is the volume fraction of counter clockwise $90^{\circ}$ switching.

When the domain only switches by $180^{\circ}$, we have

$\rho_{1}=\frac{\hat{W}_{\mathrm{cr}}^{(1)}}{A_{11}}$.

When the domain only switches by clockwise $90^{\circ}$, we have

$\rho_{2}=\frac{\hat{W}_{\mathrm{cr}}^{(2)}}{A_{22}}$.

When the domain only switches by counter clockwise $90^{\circ}$, we have

$\rho_{3}=\frac{\hat{W}_{\mathrm{cr}}^{(3)}}{A_{33}}$.

When the domain switches by both $180^{\circ}$ and clockwise $90^{\circ}$, we have

$\rho_{1}=\frac{\hat{W}_{\mathrm{cr}}^{(1)} A_{22}-\hat{W}_{\mathrm{cr}}^{(2)} A_{12}}{A_{11} A_{22}-A_{12} A_{21}}$,
$\rho_{2}=\frac{\hat{W}_{\mathrm{cr}}^{(2)} A_{11}-\hat{W}_{\mathrm{cr}}^{(1)} A_{21}}{A_{11} A_{22}-A_{12} A_{21}}$.

When the domain switches by both $180^{\circ}$ and counter clockwise $90^{\circ}$, we have

$\rho_{1}=\frac{\hat{W}_{\mathrm{cr}}^{(1)} A_{33}-\hat{W}_{\mathrm{cr}}^{(3)} A_{13}}{A_{11} A_{33}-A_{13} A_{31}}$,
$\rho_{3}=\frac{\hat{W}_{\mathrm{cr}}^{(3)} A_{11}-\hat{W}_{\mathrm{cr}}^{(1)} A_{31}}{A_{11} A_{33}-A_{13} A_{31}}$. 
If the whole domain has completely switched, but a partial domain switched by $90^{\circ}$ and the others switched by $180^{\circ}$, it means that

$\rho_{1}+\rho_{2}=1$.

It must be pointed that Eq. (31) is obtained when the mixture domain switching occurs by both $180^{\circ}$ and clockwise $90^{\circ}$. For this saturated situation of the mixture domain switching, the variation of the total energy is no longer Eq. (16), and becomes

$\sigma_{i j}\left(\Delta \gamma_{i j}^{s(2)}-\Delta \gamma_{i j}^{s(1)}\right)+E_{k}\left(\Delta D_{k}^{s(2)}-\Delta D_{k}^{s(1)}\right)+\left(\Delta d_{k i j}^{(2)}-\Delta d_{k i j}^{(1)}\right) \sigma_{i j} E_{k}=\left(W_{\mathrm{cr}}^{(2)}-W_{\mathrm{cr}}^{(1)}\right)$.

From the first one of Eqs. (4), we can get

$\sigma_{i j}=C_{i j m n}\left[\gamma_{m n}-\gamma_{m n}^{s(0)}-\left(d_{k m n}^{(0)}+\sum_{I=1}^{2} \rho_{I} \Delta d_{k m n}^{(I)}\right) E_{k}-\sum_{I=1}^{2} \rho_{I} \Delta \gamma_{m n}^{s(I)}\right]$.

Substitute Eq. (33) into the Eq. (32), so

$$
\begin{aligned}
C_{i j m n} & {\left[\gamma_{m n}-\gamma_{m n}^{s(0)}-\left(d_{k m n}^{(0)}+\sum_{I=1}^{2} \rho_{I} \Delta d_{k m n}^{(I)}\right) E_{k}-\sum_{I=1}^{2} \rho_{I} \Delta \gamma_{m n}^{s(I)}\right]\left(\Delta \gamma_{i j}^{s(2)}-\Delta \gamma_{i j}^{s(1)}\right) } \\
& +E_{k}\left(\Delta D_{k}^{s(2)}-\Delta D_{k}^{s(1)}\right) \\
& +C_{i j m n}\left(\Delta d_{k i j}^{(2)}-\Delta d_{k i j}^{(1)}\right) E_{k}\left[\gamma_{m n}-\gamma_{m n}^{s(0)}-\left(d_{k m n}^{(0)}+\sum_{I=1}^{2} \rho_{I} \Delta d_{k m n}^{(I)}\right) E_{k}-\sum_{I=1}^{2} \rho_{I} \Delta \gamma_{m n}^{s(I)}\right] \\
= & \left(W_{\mathrm{cr}}^{(2)}-W_{\mathrm{cr}}^{(1)}\right) .
\end{aligned}
$$

Then we have

$\rho_{2}=\frac{\hat{W}_{\mathrm{cr}}^{(2)}-\hat{W}_{\mathrm{cr}}^{(1)}-\left(A_{12}-A_{11}\right)}{\left(A_{11}+A_{22}\right)-A_{12}-A_{21}}, \quad \rho_{1}=1-\rho_{2}$.

\section{The calculation method}

\subsection{Finite element formulation}

The finite element formulation of $\mathrm{Li}$ and Fang [55] is employed in this paper. The displacement and electric potential are taken to be the nodal degrees of freedom in the present finite element method. The interaction between adjust domains is taken into account.

\subsection{Boundary conditions}

In Lynch's experiment [59], samples were cut to $10 \mathrm{~mm}$ cubes. Hence strictly speaking, the problem is truly $3 \mathrm{D}$. But the $3 \mathrm{D}$ finite element simulation costs too much computation time and the efficiency of the 3D finite element simulation is quite lower due to the complex distribution of the polarization direction. For simplicity a 2D finite element model was proposed and depicted in Fig. 1, in this paper.

The mechanical boundary conditions are that the $x$-displacement and the $z$-displacement at $(x=-a / 2, z=-a / 2)$ are fixed at zero. The $z$-displacement on point $(x=a / 2, z=-a / 2)$ is zero in order to prevent rigid body motion. Uniformly distributed compressive stress is applied to the 


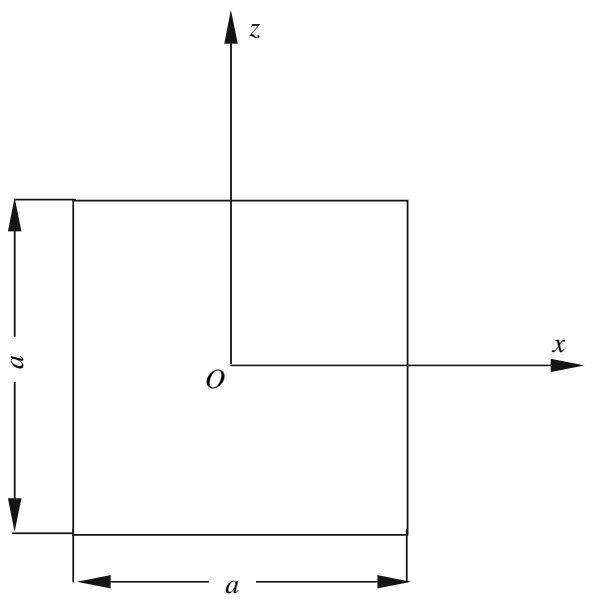

Fig. 1. The 2D finite element model

specimen on $z= \pm a / 2$. All other surfaces are free of traction. The electrical potential has the value $\phi$ on the upper surface electrode $z=+a / 2$ and is $-\phi$ on the surface $z=-a / 2$. On all other surfaces, the free charge and the normal component of the electric displacement are zero.

\subsection{The calculation method}

The samples of the polycrystalline ferroelectric ceramic were first poled by applying an electric field excess of the coercive field, this changes the original direction of the polarization vector of the domains to that most closely aligned with the positive direction of $z$-axis. Hence the polarization vectors of the domains are assigned nearly to the $z$ direction in this paper. Let $\varphi$ be the angle between the initial polarization vector of the domain and the $z$-axis. So the value of $\varphi$ is between $-\varphi_{\max }$ and $\varphi_{\max }$. The simulation is carried out with a large number of domains. All the calculations are carried out with total 12,800 elements. Each domain is represented by one element in finite element simulation. Triangle elements with uniform strain and electric field are used. The sketch of the grids is shown in Fig. 2. The macroscopic response of the specimen is computed from the volume average response of each domain,

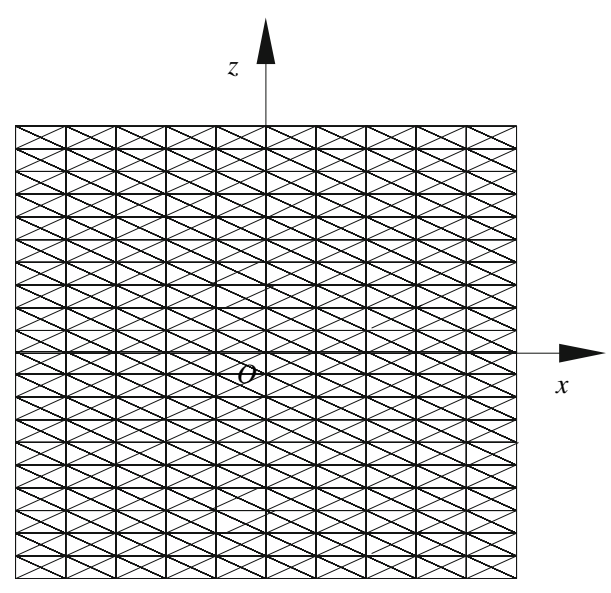

Fig. 2. The sketch of finite element calculation 
$\gamma_{i j}=\frac{1}{V}\left(\sum_{l=1}^{N} \gamma_{i j(l)} V_{l}\right)$,

$D_{i}=\frac{1}{V}\left(\sum_{l=1}^{N} D_{i(l)} V_{l}\right)$,

where $N_{t}$ is the total number of the triangle elements, $V$ is the volume of the ferroelectric body, $V_{l}$ is the volume of the $l$-th element, $\gamma_{i j(l)}, D_{i(l)}$ is the strain and electric displacement of the $l$-th element, respectively.

Polycrystalline ferroelectric ceramic (8/65/35 PLZT) was used in the experiment studied by Lynch [59]. The material parameters are $\mathrm{Y}=68 \times 10^{9} \mathrm{~Pa}, v=0.3, \varepsilon_{33}=56.25 \times 10^{-9} \mathrm{C} /(\mathrm{V} \mathrm{m})$, $P_{r}=0.25 \mathrm{C} / \mathrm{m}^{2}, \gamma_{r}=0.143 \%, E_{c}=0.36 \mathrm{MV} / \mathrm{m}$.

The interaction among domains is naturally taken account in the finite element simulation. Because different elements have different spontaneous polarizations and spontaneous strains, the internal stress and electric field generate due to polarizations and strain incompatibilities, when the external electric field and mechanical loads are zero. One can get the following relations:

$P_{r}=\alpha P_{s}$,

$\gamma_{r}=\beta \gamma_{s}$.

Hence one can obtain the suitable parameters $P_{s}$ and $\gamma_{s}$ which guarantee the remnant polarization $P_{r}=0.25 \mathrm{C} / \mathrm{m}^{2}$ and $\gamma_{r}=0.143 \%$. In this paper $\alpha$ and $\beta$ are taken to be 0.953 and 0.98 , respectively, for the case of $\varphi_{\max }=15^{\circ}$. The material constants and parameters in the present simulation are chosen to be $\mathrm{Y}=50 \times 10^{9} \mathrm{~Pa}, v=0.3, \varepsilon_{33}=62 \times 10^{-9} \mathrm{C} /(\mathrm{V} \mathrm{m}), P_{s}=0.254 \mathrm{C} / \mathrm{m}^{2}, \gamma_{s}=0.15 \%$, $E_{c}=0.36 \mathrm{MV} / \mathrm{m}, d_{33}=0.9 \times 10^{-9} \mathrm{~m} / \mathrm{V}, d_{31}=-4.5 \times 10^{-10} \mathrm{~m} / \mathrm{V}, d_{15}=1.545 \times 10^{-9} \mathrm{~m} / \mathrm{V}$.

\subsection{The hardening function}

The experimental results by Lynch [59] have clearly shown that the critical energy barrier $W_{\mathrm{cr}}^{(I)}$ will increase when the volume fraction $\rho_{I}$ of the domain switching increases. In other words, the driving force of domain switch which equals the critical energy barrier $W_{\mathrm{cr}}^{(I)}$ will be hardening as the domain switching processes.

Li and Weng [8], [9] were the first to introduce the concept of a hardening relation for ferroelectric materials in their several papers. They thought the energy dissipation associated with the movement of domain walls will increase with increasing domain concentration and assumed a simple nonlinear function with several unknown parameters which needed to be determined by comparison of the calculation results with the experiment results for purely mechanical loading or purely electric loading.

In the present paper, the hardening relation between the driving force of domain switch and the volume fraction $\rho_{I}$ of domain switching is calibrated based on the partial experimental results for the poled ferroelectric ceramic specimen. The detailed procedure is given by Liu et al. [60].

The non-dimensional hardening relation can be written as

$$
\begin{aligned}
& f_{1}\left(\rho_{1}\right)=W_{\mathrm{cr}}^{(1)} / 2 E_{c} P_{s}, \\
& f_{2}\left(\rho_{2}\right)=W_{\mathrm{cr}}^{(2)} / 1.5 \sigma_{c} \gamma_{s}^{s} .
\end{aligned}
$$


The calibrated results for polycrystalline ferroelectric ceramic (8/65/35 PLZT) under combined mechanical and electric loading are listed as follows:

(i) The hardening function for $90^{\circ}$ domain switching is expressed as

$$
f_{2}\left(\rho_{2}\right)=\frac{7.340 \rho_{2}}{0.2790+\rho_{2}}+\frac{1.835 \rho_{2}}{0.9799-\rho_{2}}-6.256 \rho_{2}+1
$$

(ii) The hardening function for $180^{\circ}$ domain switch is

$$
f_{1}\left(\rho_{1}\right)=E_{c}^{\sigma} / E_{c}+B \cdot \rho_{1},
$$

where $E_{c}^{\sigma}$ is the coercive electric field under a suppressive stress $\sigma$,

$$
B=0.0242-0.3607 n_{i} \sigma_{i j} n_{j} / \sigma_{c} .
$$

Generally speaking, the ferroelectric ceramics contain numerous internal micro voids, hence there is an additional nonlinear deformation when the specimen of the ferroelectric ceramics is subjected to compressive loading. We call this additional strain as plastic strain. According to the experimental result for purely mechanical loading by Lynch [59], the overall plastic strain for the specimen can be expressed as

$\gamma_{33}^{p}=0.01477\left(e^{-0.5856 \sigma_{33} / \sigma_{c}}-1\right), \quad 1<-\sigma_{33} / \sigma_{c}<3.57$,

$\gamma_{33}^{p}=-0.125 \%, \quad-\sigma_{33} / \sigma_{c} \geq 3.57$.

The domain switching criterion used in this paper is

$E_{i} \Delta D_{i}^{s(I)}+\lambda \hat{\sigma}_{11} \Delta \hat{\gamma}_{11}^{s(I)}+\hat{\sigma}_{33} \Delta \widehat{\gamma}_{33}^{s(I)}=W_{\mathrm{cr}}^{(I)}$,

where $\hat{\sigma}_{11}$ and $\hat{\sigma}_{33}$ are the stresses in the local coordinate system $\hat{x}_{j}$ aligned with the crystal axes of the initial single domain and $\Delta \hat{\gamma}_{11}^{s(I)}, \Delta \hat{\gamma}_{33}^{s(I)}$ are the corresponding items in the local coordinate system $\hat{x}_{j}$.

The internal stresses induced as a result of the incompatibility spontaneous strains are much higher than the critical stress $\sigma_{c}$, so that the second term of the driving force is modified by introducing a modification factor $\lambda$. If $\lambda=1$, the domain switching criterion (47) is completely consistent with Hwang's criterion. In the present simulation the modification factor $\lambda$ is taken to be 0.8 . The applied field is gradually increased from zero to maximum, and then reversed. The volume fraction $\rho_{I}$ of domain switching for each element obtained from the last loading step is used as input to the constitutive equations. The strain and electric fields are calculated based on the finite element procedure. Using the computed strain and electric fields, the new values of the volume fraction $\rho_{I}$ of domain switching are obtained from Eq. (19).

\section{The calculated results}

\subsection{Uniaxial mechanical loading}

The nonlinear constitutive behavior of the ferroelectric specimen subjected to a purely uniaxial compressive stress is studied in this Section. 
Divide the load $-85 \mathrm{MPa}$ into 100 steps. The external mechanical loading is increased step by step. Figures 3 and 4 show the curves of the stress versus electric displacement and stress versus strain.

The solid lines in Figs. 3 and 4 correspond to the experimental results by Lynch [59]. The present FEM results are denoted by the open triangles.

In our simulation, we find that a lot of domain switches before the compressive stress is applied on the specimen. Those switchings are induced by large internal stress and electric field. But most of the calculated volume fractions of domain switching are quite small. When the load is gradually increased, the calculated volume fraction is increased simultaneously. Since most of the calculated volume fractions of domain switching are still small, the behavior of the stress-electric displacement and stress-strain is nearly linear at beginning from 0 to $-10 \mathrm{MPa}$.

At $\sigma_{33}=-15 \mathrm{MPa}$, in about $60 \%$ of all elements the volume fraction of $90^{\circ}$ domain switching is greater than 0.1 . At $\sigma_{33}=-30 \mathrm{MPa}$, in about $80 \%$ of all elements the volume fraction of $90^{\circ}$ domain switching is greater than 0.4 . At $\sigma_{33}=-85 \mathrm{MPa}$, in about $40 \%$ of all elements the volume fraction of $90^{\circ}$ domain switching is greater than 0.8 and in another about $40 \%$ of total elements the volume fraction of $90^{\circ}$ domain switching is within the range $0.4 \sim 0.8$. Figure 3 shows that the calculated electric displacement of the specimen is greater than the experimental results for the case $\left|\sigma_{33}\right|<45 \mathrm{MPa}$. At $-85 \mathrm{MPa}$ the calculated electric displacement $D_{3}$ is less than the experimental result. Figure 4 shows the axial stress-strain curve. When $\left|\sigma_{33}\right|$ is less than $30 \mathrm{MPa}$, the calculated axial strain is in good agreement with the experimental result. With the increasing of the compressive stress the calculated axial strain $\gamma_{33}$ deviates the experimental curve gradually. The reason may be described as follows. The orientation angle $\varphi$ between the direction of polarization and the $z$-axis is zero in the mechanical model of the single domain (see Appendix B). But the orientation angle $\varphi$ of each domain is distributed between $-\varphi_{\max }$ and $\varphi_{\max }$ in the present finite element simulation. Hence in the same loading, the driving force in the mechanical model is greater than that in the finite element analysis. In the same way, the volume fraction of domain switching in the mechanical model is greater than that in the finite element analysis.

\subsection{Uniaxial electric field loading}

Figures 5 and 6 show the ferroelectric hysteresis and butterfly shaped curve under the uniaxial electric loading, respectively. First we discuss the negative electric field loading ( $E_{3}$ opposite to the

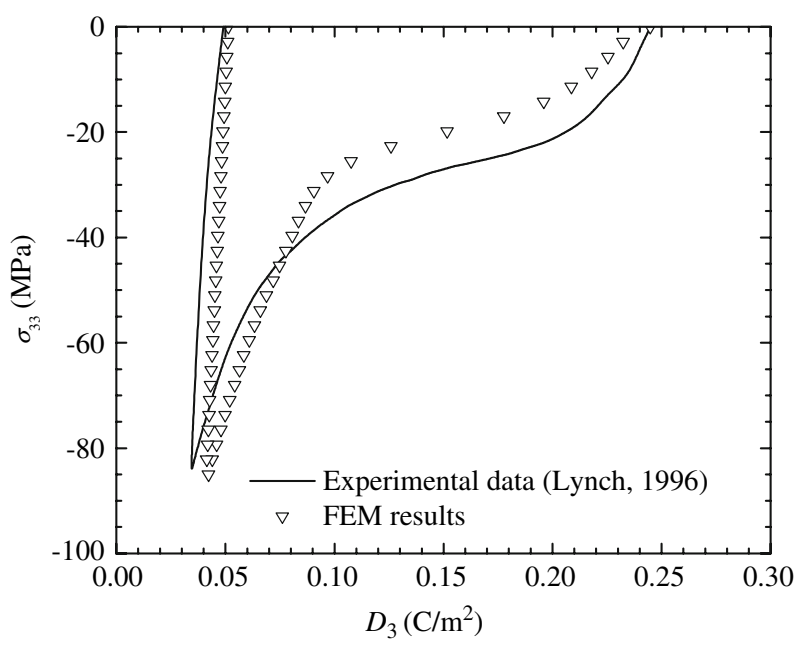

Fig. 3. Simulated and measured stress vs. electric displacement at zero applied electric field 

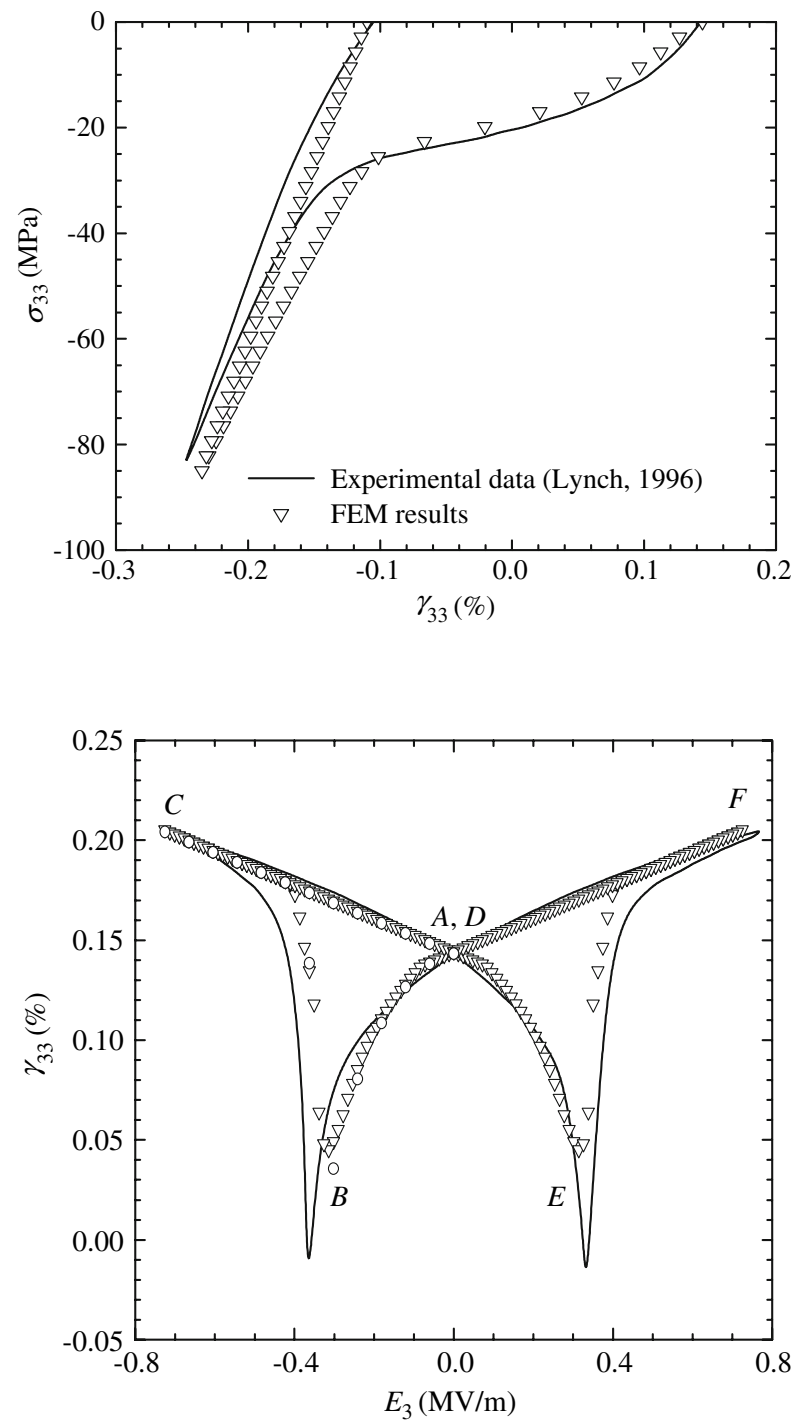

Fig. 4. Simulated and measured stress vs. strain at zero applied electric field
Fig. 5. Comparison of simulated and measured strain vs. electric field curves at $\sigma_{33}=0$

direction of the remanent polarization of the specimen). From Fig. 5 we can see that the calculated axial strain $\gamma_{33}$ agrees well with the experimental results from point $A$ to point $B$. The strain of the poled ceramic decreases linearly due to the piezoelectric effect as the electric load is lower; then, the strain drops sharply and reaches the minimum at point $B$ as depolarization occurs. The strain then rises to reach saturation at point $C$ after repolarization. As polarization reversal takes place, the piezoelectric coefficients of the ferroelectric specimen change their signs. This leads to a reversal of the macroscopic piezoelectric effect, manifested in the change of slope in the linear regime from positive to negative. The strain decreases linearly due to the piezoelectric effect as the negative electric field is gradually decreased from point $C$.

As shown in Fig. 6 the calculated electric displacement $D_{3}$ is in reasonable agreement with the experimental results before $E_{c}$. At $-0.1 \mathrm{MV} / \mathrm{m}$, the volume fraction of $90^{\circ}$ domain switching of all elements is larger than 0.1 . When the electric field is less than $-0.30 \mathrm{MV} / \mathrm{m}$, the elements of $0<\rho$ ( $\rho=\rho_{2}$ or $\left.\rho_{3}\right) \leq 0.4$ decrease quickly, and the elements of $0.4<\rho \leq 0.8$ increase quickly. But when the electric field is less than $-0.36 \mathrm{MV} / \mathrm{m}$, the elements of $0<\rho \leq 0.4$ increase quickly, and 


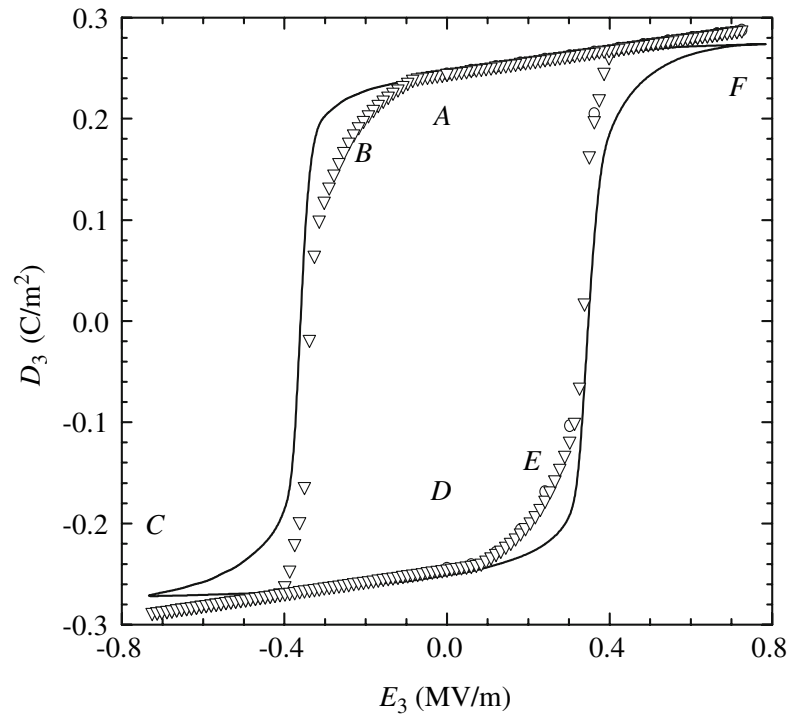

Fig. 6. Comparison of simulated and measured electric displacement versus electric field curves at $\sigma_{33}=0$

the elements of $0.4<\rho \leq 0.8$ decrease quickly because the $180^{\circ}$ domain switching takes place. At $-0.44 \mathrm{MV} / \mathrm{m}$, the volume fraction $\rho_{1}$ of $180^{\circ}$ domain switching of all elements is larger than 0.8 . Now a positive electric field is applied to the specimen from point $D$. With the electric field $E_{3}$ increasing, the domain switching occurs in most of all elements. The situation of the domain switching is similar to that of the negative electric loading from point $A$ to points $B$ and $C$. The computed strain $\gamma_{33}$ and electric displacement $D_{3}$ for the specimen agree well with the experimental data from point $D$ to point $E$ and point $F$. The computed strain $\gamma_{33}$ and electric displacement $D_{3}$ for the specimen match well with the experimental data for unloading from point $F$ and point $A$.

\subsection{Combined electromechanical loading}

As for electromechanical coupling loading, first a prescribed compressive stress is applied to the specimen. Then a circle of electric field is applied to the specimen while the external compressive stress is held fixed. The open triangles in Figs. 7 and 12 show the results of the calculated electric displacement and strain versus the applied electric field with different compressive stress loading. In the current simulation the switching process under applied compressive stress is more gradual than that at zero stress. The solid lines in Figs. 7-12 correspond to the experimental results obtained by Lynch [59].

First we discuss the case of $\sigma_{33}=-15 \mathrm{MPa}$. This prescribed compressive stress is applied to the specimen and held fixed. An electric field parallel to the applied stress is then introduced and cycled between positive and negative limits. At zero electric field, the volume fraction of $90^{\circ}$ domain switching of all elements is larger than 0.1 , because the applied compression encourages the domain switching. When the negative electric field increases, the elements of $0.4<\rho \leq 0.8$ also increase slowly. Until $-0.15 \mathrm{MV} / \mathrm{m} 180^{\circ}$ domain switches take place. At maximum electric field, the volume fraction $\rho_{1}$ of $180^{\circ}$ domain switching of all elements is larger than 0.8 , so when unloading the electro-mechanical response is linear. The general feature of the hysteresis loop for the applied stress of $-15 \mathrm{MPa}$ is captured in the predicted curve as shown in Fig. 8. But the calculated butterfly loop is remarkably deviating from the experimental result as shown in Fig. 7.

For the cases of $\sigma_{33}=-30 \mathrm{MPa}$, the switch process is similar to that of $\sigma_{33}=-15 \mathrm{MPa}$. At zero electric field, the volume fraction of the $90^{\circ}$ domain switching of almost all elements is 

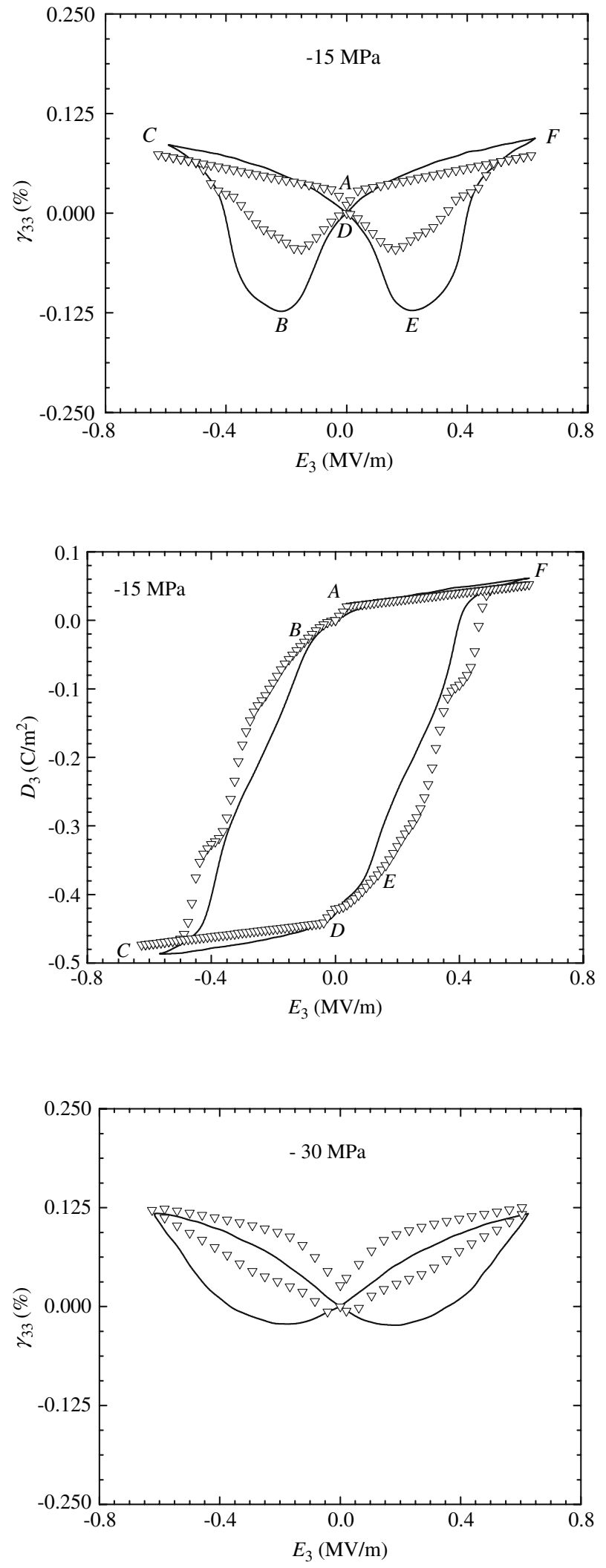

Fig. 7. Comparison of simulated and measured strain vs. electric field curves at $\sigma_{33}=-15 \mathrm{Mpa}$

Fig. 8. Comparison of simulated and measured electric displacement vs. electric field curves at $\sigma_{33}=-15 \mathrm{Mpa}$
Fig. 9. Comparison of simulated and measured strain vs. electric field curves at $\sigma_{33}=-30 \mathrm{Mpa}$ 

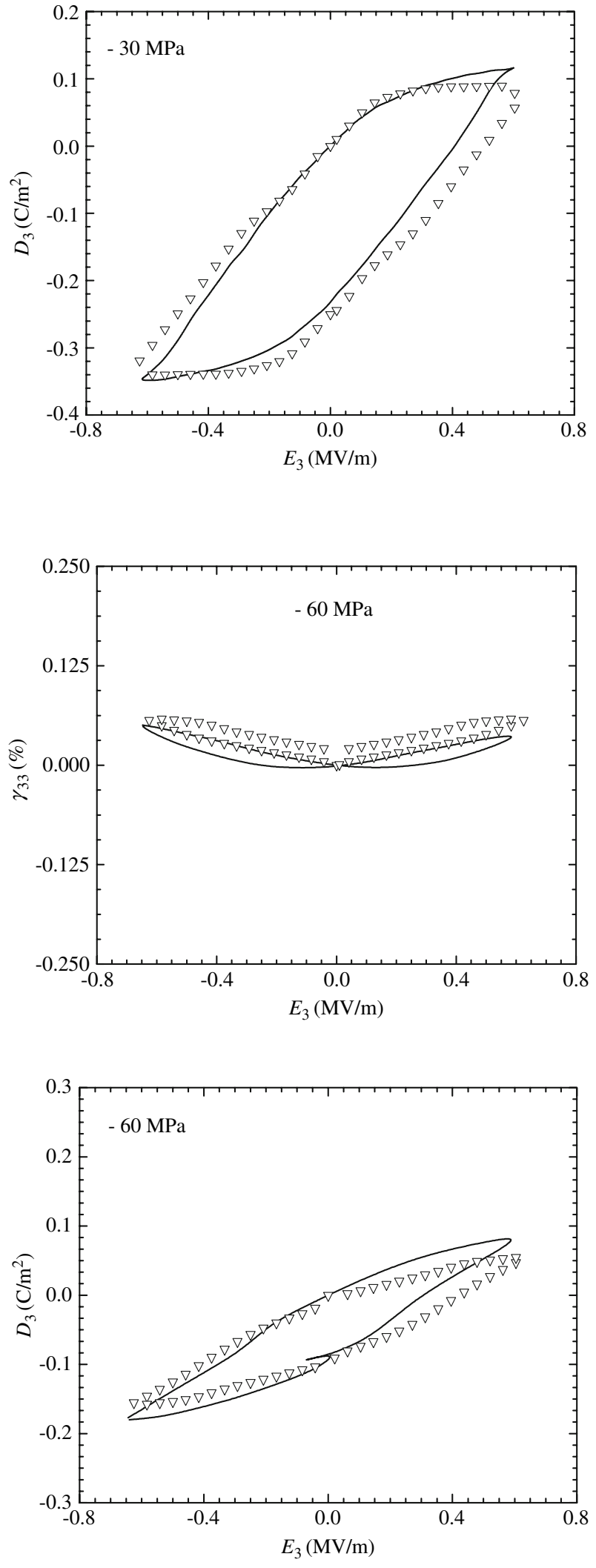

Fig. 10. Comparison of simulated and measured electric displacement vs. electric field curves at $\sigma_{33}=-30 \mathrm{Mpa}$
Fig. 11. Comparison of simulated and measured strain vs. electric field curves at $\sigma_{33}=-60 \mathrm{Mpa}$
Fig. 12. Comparison of simulated and measured electric displacement vs. electric field curves at $\sigma_{33}=-60 \mathrm{Mpa}$ 
larger than 0.4. When the negative electric field increases, the elements of $0.4<\rho \leq 0.8$ are decreasing linearly. At $-0.05 \mathrm{MV} / \mathrm{m}$, the $180^{\circ}$ domain switches take place. After that the elements of $0.8<\rho_{1} \leq 1.0$ increase linearly and the elements of $0.4<\rho \leq 0.8$ decrease linearly as the negative electric field increases. The calculated hysteresis loop for the applied stress of $-30 \mathrm{MPa}$ agrees well with the experimental results as shown in Fig. 10, but the calculated butterfly loop is obviously deviating from the experimental results as shown in Fig. 9.

For the case of $\sigma_{33}=-60 \mathrm{MPa}$, the volume fraction of $90^{\circ}$ domain switching of all elements is larger than 0.4 at zero electric field. When the negative electric field increases, the elements of $0.4<\rho \leq 0.8$ decrease linearly and the elements of $0.8<\rho \leq 1.0$ increase linearly. From -0.05 to $-0.36 \mathrm{MV} / \mathrm{m}$, the elements of $0.4<\rho \leq 0.8$ and $0.8<\rho \leq 1.0$ decrease slightly, meanwhile the elements of $0.0<\rho_{1} \leq 0.8$ increase linearly. The general feather of the calculated butterfly loop for the applied stress of $-60 \mathrm{MPa}$ agrees well with the experimental curve as shown in Fig. 11. But the hysteresis loop is obviously deviating from the experimental results when $E_{3}>$ $0.2 \mathrm{MV} / \mathrm{m}$ as shown in Fig. 12. This deviation is probably associated with a simplification of the model in handing the relation of 180 switching. A simple linear fit of the hardening relation of 180 switching cannot reflect the actual evolution process of the domain wall.

\section{Conclusions}

The principle of stationary total potential energy is put forward in this paper. The mechanical field equation and a domain switching criterion are deduced from the energy principle. The domain switching criterion established in this paper is the expansion and development of the energy criterion established by Hwang et al. [1]. The 2D finite element procedure for domain switching in ferroelectric specimens is developed. The hardening relation between the driving force of domain switching and the volume fraction of domain switching is calibrated based on the single domain mechanical model and partial experimental results. Using this calibrated hardening relation, the nonlinear response of the ferroelectric specimen is calculated. A systematic finite element analysis was performed on the nonlinear behavior of ferroelectric ceramics subjected to the electromechanical coupling loading. The results involve the electric butterfly shaped curves of axial strain versus axial electric field, the hysteresis loops of the electric displacement versus electric field and the evolution process of the domain switching in the ferroelectric specimen under uniaxial coupled stress and electric field. The present theoretic prediction for hysteresis loops agrees reasonably with the experimental results given by Lynch [59]. The simulated butterfly loops agree well with experimental results [50] for purely mechanical loading and purely electric loading, but for combined electromechanical loading the present predictions for hysteresis loops are obviously deviating from the experimental results [50] which needs to be studied in the future.

\section{Appendix A}

The total energy $\Phi$ can be expressed as

$\Phi=\int_{V} U d V-\int_{S_{\sigma}} \bar{t}_{i} u_{i} d S+\int_{S_{\phi}} \bar{\phi} D_{i} n_{i} d S+\int_{V}\left(\sum_{I=1}^{N} \rho_{I} W_{c r}^{(I)}\right) d V$.

Using the Lagrange multiplier $\lambda$, the principle of stationary total energy $\Phi$ is equivalent to the following principle of stationary functional $\tilde{\Phi}$ : 
$\tilde{\Phi}=\Phi-\int_{V} \lambda D_{i, i} d V$

The first variation of $\tilde{\Phi}$ has the form

$$
\begin{aligned}
\delta \tilde{\Phi}= & \int_{V}\left(\sigma_{i j} \delta \gamma_{i j}+E_{i} \delta D_{i}\right) d V-\int_{S_{\sigma}} \bar{t}_{i} \delta u_{i} d S+\int_{S_{\phi}} \bar{\phi} n_{i} \delta D_{i} d S \\
& +\int_{V}\left(\sum_{I=1}^{N} \delta \rho_{I} W_{c r}^{(I)}\right) d V-\int_{V} D_{i, i} \delta \lambda d V-\int_{V} \lambda \delta D_{i, i} d V .
\end{aligned}
$$

The above equation can be rewritten as

$$
\begin{aligned}
\delta \tilde{\Phi}= & \int_{v}\left[-\sigma_{i j, j} i+\left(E_{i}+\lambda_{, i}\right) \delta D_{i}\right] d V+\int_{S_{\sigma}}\left(\sigma_{i j} n_{j}-\bar{t}_{i}\right) \delta u_{i} d S-\int_{s_{\phi}}(\lambda-\bar{\phi}) n_{i} \delta D_{i} d s \\
& -\int_{v} \sum_{I=1}^{N} \delta \rho_{I}\left(\sigma_{i j} \Delta \gamma_{i j}^{S(I)}+E_{k} \Delta D_{k}^{S(I)}+\frac{1}{2} \Delta S_{i j m n}^{(I)} \sigma_{m n} \sigma_{i j}+\frac{1}{2} \Delta \varepsilon_{i k}^{(I)} E_{k} E_{i}+\Delta d_{k i j}^{(I)} \sigma_{i j} E_{k}-W_{c r}^{(I)}\right) d V \\
& -\int_{v} D_{i, i} \delta \lambda d V .
\end{aligned}
$$

The necessary condition for $\tilde{\Phi}$ to be stationary is

$\delta \tilde{\Phi}=0$.

From Eq. (A.5), one can obtain

$\sigma_{i j, j}=0$,

$E_{i}=-\lambda, i$. in $V$,

$D_{i, i}=0$,

$\sigma_{i j} \Delta \gamma_{i j}^{s(I)}+E_{k} \Delta D_{k}^{s(I)}+\frac{1}{2} \Delta S_{i j m n}^{(I)} \sigma_{m n} \sigma_{i j}+\frac{1}{2} \Delta \varepsilon_{i k}^{(I)} E_{k} E_{i}+\Delta d_{k i j}^{(I)} \sigma_{i j} E_{k}=W_{c r}^{(I)}$ in $V$,

$\sigma_{i j} n_{j}=\bar{t}_{i}, \quad$ on $S_{\sigma}$,

$\lambda=\bar{\phi}, \quad$ on $S_{\phi}$.

Clearly these are the basic equations and the boundary conditions for the ferroelectric body. From Eqs. (A.6) and (A.9) one can see that the Lagrange multiplier $\lambda$ is the electric potential $\phi$.

\section{Appendix B}

Mechanical model

In order to describe the electromechanical coupling phenomenon of the ferroelectric specimen, the hardening relation between the driving force of domain switching and the volume fraction of domain switching needs to be determined.

A plane strain single domain mechanical model is proposed in this Appendix. The poled ferroelectric specimen is considered as a transversely isotropic homogeneous material with single domain. The single domain is assumed to switch when the reduction of potential energy of the 
system due to that switch exceeds a critical value, which can be considered as the energy barrier that must be overcome to achieve the switch.

The switching criterion proposed by Hwang et al. [1] can be expressed as

$E_{i} \Delta D_{i}^{s(I)}+\sigma_{i j} \Delta \gamma_{i j}^{s(I)}=W_{c r}^{(I)}$.

The initial energy barrier against the $90^{\circ}$ switch is assumed to be $3 \sigma_{c} \gamma^{s} / 2$, the initial energy barrier against the $180^{\circ}$ switch is $2 E_{\mathrm{c}} P_{\mathrm{s}}, \sigma_{\mathrm{c}}$ is the critical stress, $\gamma^{s}$ is the spontaneous strain.

From the experimental results by Lynch [50], one can see that the butterfly loop tails become narrow and short when the compressive stress increases. The narrow tails indicate that the coercive electric fields $E_{\mathrm{c}}^{\sigma}$ reduce with the compressive stress increasing clearly.

Suppose the volume fraction of $180^{\circ}$ switching is $\rho_{1}$. The volume fraction of clockwise $90^{\circ}$ switching can be expressed as $\rho_{2}$. The volume fraction of counter clockwise $90^{\circ}$ switching can be expressed as $\rho_{3}$. Due to the symmetry of the model, only clockwise $90^{\circ}$ switch is considered in this model. The whole process of domain switching contains four stages when the ferroelectric specimen is subjected to both the negative electric and compressive mechanical loading. First the volume fraction $\rho_{2}$ of $90^{\circ}$ switching increases when $90^{\circ}$ domain switching takes place. Then the $180^{\circ}$ switching occurs when the applied electric field reaches the coercive electric field $E_{\mathrm{c}}^{\sigma}$, meanwhile the $\rho_{2}$ keeps constant since the driving force of domain switch for the second $90^{\circ}$ switching is less than the energy barrier. Third when $\rho_{1}+\rho_{2}$ reaches $1, \rho_{1}$ increases continuously and $\rho_{2}$ decreases to keep $\rho_{1}+\rho_{2}=1$. Finally, the electric field is reduced to zero while the mechanical load keeps constant. According to this process of domain switching, the hardening relation between the driving force of domain switching and the volume fraction of domain switching can be calibrated using the partial experimental data by Lynch [50].

\section{Purely mechanical loading}

According to the domain switching criterion, only $90^{\circ}$ domain switching occurs. Using the constitutive equation (4) one can obtain

$\rho_{2}=\frac{D_{3}-D_{3}^{s(0)}-d_{333}^{(0)} \sigma_{33}}{\Delta D_{3}^{s(2)}+\Delta d_{333}^{(2)} \sigma_{33}}$,

where $D_{3}$ is the experimental result for the ferroelectric specimen under purely mechanical loading.

\section{Electromechanical loadings}

After a prescribed compressive stress is applied to the specimen and held fixed, the strain of the specimen can be expressed as $\gamma_{33}^{(0)}$, and the volume fraction of $90^{\circ}$ domain switching is $\rho_{2}^{(0)}$. Then a circle of electric field is introduced. The strain increment is

$\Delta \gamma_{33}=\gamma_{33}-\gamma_{33}^{(0)}=\left(\rho_{2}-\rho_{2}^{(0)}\right) \Delta \gamma_{33}^{s(2)}+\left(d_{333}^{(0)}+\rho_{1} \Delta d_{333}^{(1)}+\rho_{2} \Delta d_{333}^{(2)}\right) E_{3}$.

Only the $90^{\circ}$ domain switch occurs when the applied electric field is less than the coercive electric field $E_{c}^{\sigma}$,

$\rho_{2}=\frac{\Delta \gamma_{33}+\rho_{2}^{(0)} \Delta \gamma_{33}^{s(2)}-d_{333}^{(0)} E_{3}}{\Delta \gamma_{33}^{s(2)}+\Delta d_{333}^{(2)} E_{3}}$. 
After the applied electric field is larger than the coercive electric field $E_{c}^{\sigma}$, the $180^{\circ}$ domain switch occurs. If $\rho_{1}+\rho_{2}$ are less than $1, \rho_{1}$ increases continuously and $\rho_{2}$ keeps constant,

$\rho_{1}=\frac{\Delta \gamma_{33}-\left(\rho_{2}-\rho_{2}^{(0)}\right) \Delta \gamma_{33}^{s(2)}-\left(d_{333}^{(0)}+\rho_{2} \Delta d_{333}^{(2)}\right) E_{3}}{\Delta d_{333}^{(1)} E_{3}}$.

When $\rho_{1}+\rho_{2}$ reaches $1, \rho_{1}$ increases continuously and $\rho_{2}$ decreases,

$\rho_{1}=\frac{\Delta \gamma_{33}-\left(1-\rho_{2}^{(0)}\right) \Delta \gamma_{33}^{s(2)}-\left(d_{333}^{(0)}+\Delta d_{333}^{(2)}\right) E_{3}}{\left(\Delta d_{333}^{(1)}-\Delta d_{333}^{(2)}\right) E_{3}-\Delta \gamma_{33}^{s(2)}}$,

$\rho_{2}=1-\rho_{1}$

\section{The hardening relation}

Based on the partial experimental results, one can easily obtain the measurement value of $\gamma_{33}$ or $D_{3}$ when the ferroelectric specimen is subjected to different stress and electric fields. Substituting the stress and electric field and the corresponding $\gamma_{33}$ or $D_{3}$ into (B.2)-(B.6), the hardening relation between the driving force of the domain switching and the volume fraction of the domain switching can be calibrated. Then the relation can be non-dimensionalized by the initial energy barrier:

$f_{1}\left(\rho_{1}\right)=W_{c r}^{(1)} / 2 E_{c} P_{s}$,

$f_{2}\left(\rho_{2}\right)=W_{c r}^{(2)} / 1.5 \sigma_{c} \gamma_{s}^{s}$.

According to the experimental data measured by Lynch [50], the corresponding "coercive electric fields" $E_{\mathrm{c}}^{\sigma}$ of ferroelectric ceramics are $0.36,0.2,0.14,0.075 \mathrm{MV} / \mathrm{m}$ under the uniaxial compressive stresses $0,-15,-30,-60 \mathrm{MPa}$, respectively.

The experimental data taken in the following analyses are based on Lynch's work [50] for ferroelectric ceramics PLZT 8/65/35.

First we discuss $90^{\circ}$ domain switching:

\section{Purely mechanical loadings}

The hardening relation of $90^{\circ}$ domain switch between the driving force of domain switching and the volume fraction of domain switching is shown in Fig. 13.

The hardening curves of $90^{\circ}$ domain switch under electromechanical coupling loadings are quite similar to those of purely mechanical loading as also shown in Fig. 13. A single function is used to fit the similar hardening relation in two loading conditions. The function can be expressed as

$f_{2}\left(\rho_{2}\right)=\frac{7.340 \rho_{2}}{0.2790+\rho_{2}}+\frac{1.835 \rho_{2}}{0.9799-\rho_{2}}-6.256 \rho_{2}+1$.

The ferroelectric ceramics contains usually many micro pores. The irreversible strain, which is called "plastic" strain, denoted as $\gamma_{33}^{p}$, will be generated under compressive loading due to the pores. According to the experimental results measured by Lynch [50], one can obtain the following fitting formula:

$\gamma_{33}^{p}=0.01477\left(\mathrm{e}^{-0.5856 \sigma_{33} / \sigma_{c}}-1\right), \quad-\sigma_{33} / \sigma_{c}<3.26$. 


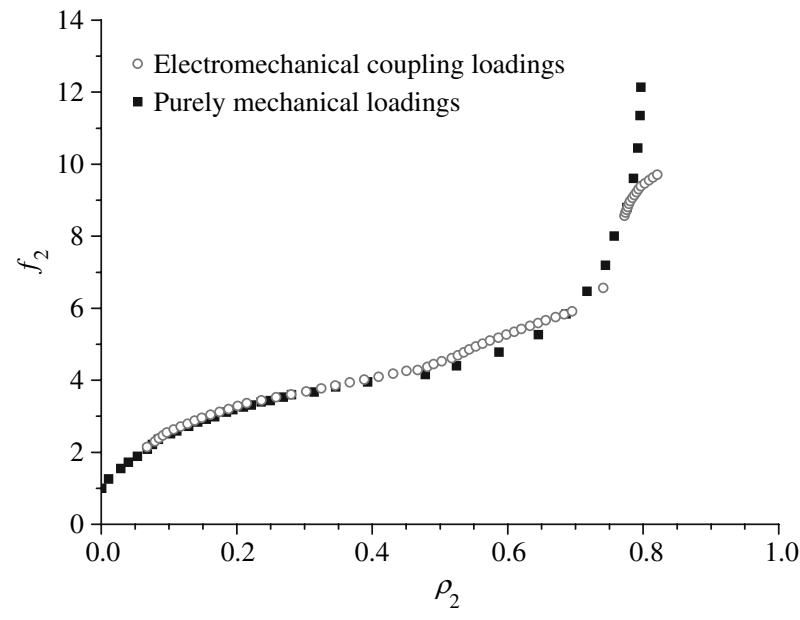

Fig. 13. The hardening relation between $f_{2}$ and $\rho_{2}$ under purely mechanical loading and combined electromechanical loadings

It must be pointed out that the formula (48) is only suitable for $-\sigma_{33} / \sigma_{\mathrm{c}}<3$. 26. When $-\sigma_{33} / \sigma_{c} \geq$ 3. 26, these pores will collapse so that the plastic strain keeps constant as shown in Fig. 14. Hence the plastic strain $\gamma_{33}^{p}$ is equal to $-0.085 \%$ for $-\sigma_{33} / \sigma_{\mathrm{c}} \geq 3.26$.

\section{The hardening relation of $180^{\circ}$ domain switch}

The hardening relation curves of $180^{\circ}$ domain switching between the driving force and the volume fraction of domain switching are shown in Fig. 15. The experimental data is complex (Fig. 16).

A linear function is used to fit the experimental data, which can be expressed by

$f_{1}\left(\rho_{1}\right)=E_{c}^{\sigma} / E_{c}+B \cdot \rho_{1}$,

where $E_{c}^{\sigma}$ is the corresponding coercive electric field of ferroelectric specimen under a suppressive stress $\sigma$. The slope $B$ versus the corresponding stress $\sigma$ displays a linear relation,

$B=0.0242-0.3607 \sigma_{33} / \sigma_{c}$.

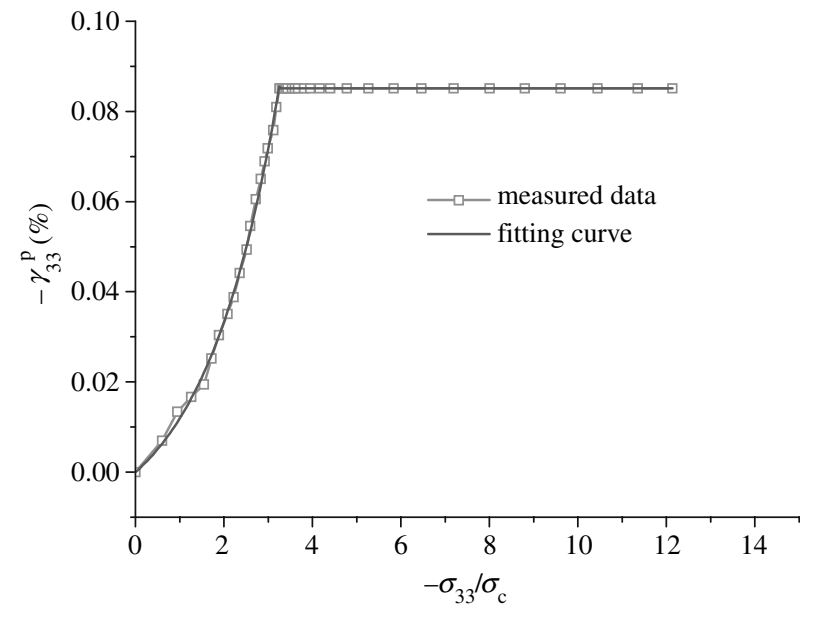

Fig. 14. The relationship and fitted curves between $\gamma_{33}^{p}$ and $-\sigma_{33} / \sigma_{c}$ 

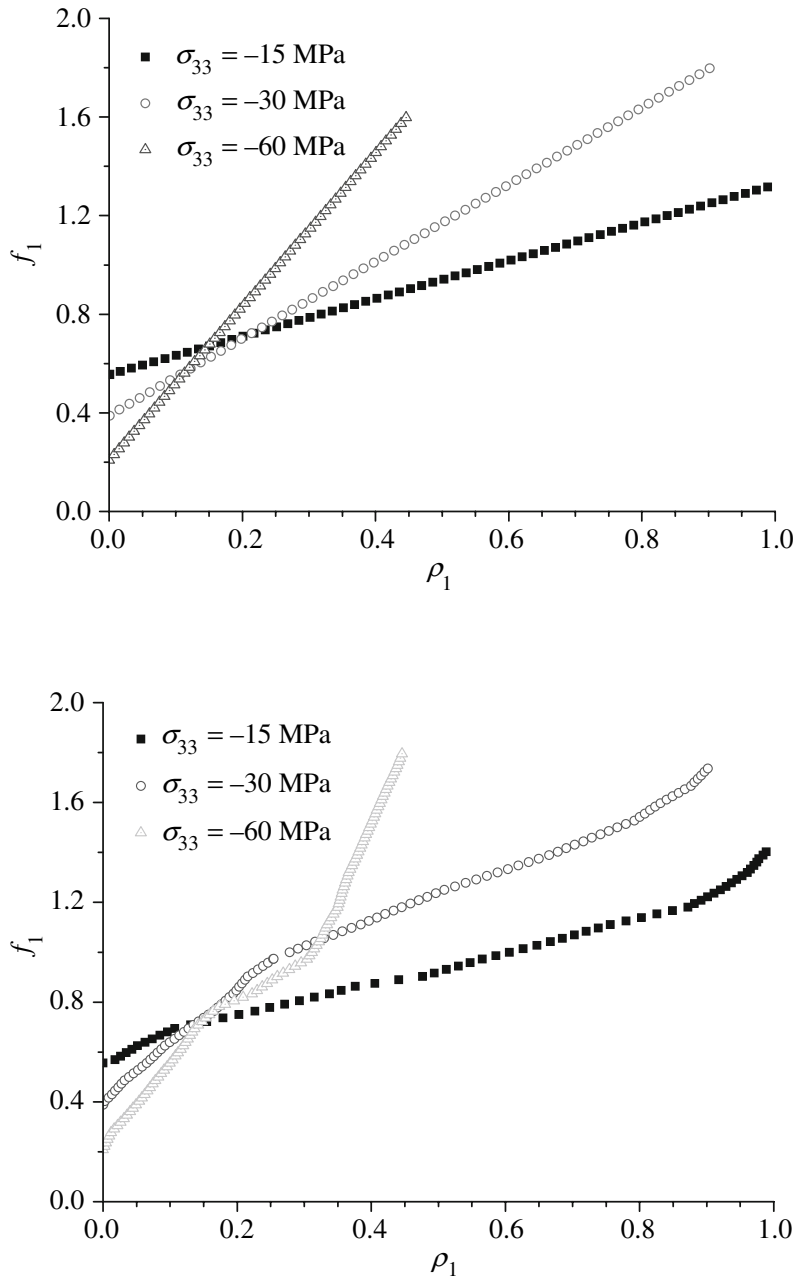

Fig. 15. The linear fitted hardening function between $f_{1}$ and $\rho_{1}$

Fig. 16. The hardening relation between $f_{1}$ and $\rho_{1}$

\section{Acknowledgments}

The present work is supported by the National Natural Science Foundation of China (No. 10572138).

\section{References}

[1] Hwang, S.C., Lynch, C.S., McMeeking, R.M.: Ferroelectric/ferroelastic interactions and a polarization switching model. Acta Metall. Mater. 43, 2073-2084 (1995)

[2] Lu, W., Fang, D.N., Hwang, K.C.: Nonlinear electric-mechanical behavior and micro-mechanics modeling of ferroelectric domain evolution. Acta Mater. 47, 2913-2926 (1999)

[3] Chen, X., Fang, D.N., Hwang, K.C.: Micromechanics simulation of ferroelectric polarization switching. Acta Mater. 45, 3181-3189 (1997)

[4] Huo, Y.Z., Jiang, Q.: Modeling of domain switching in ferroelectric ceramics: an example. Int. J. Solids Struct. 35, 1339 (1998)

[5] Huber, J.E., Fleck, N.A., Landis, C.M., McMeeking, R.M.: A constitutive model for ferroelectric polycrystals. J. Mech. Phys. Solids 47, 1663-1697 (1999)

[6] Huber, J.E., Fleck, N.A.: Multi-axial electrical switching of a ferroelectric: theory versus experiment. J. Mech. Phys. Solids. 49, 785-811 (2001) 
[7] Huber, J.E., Fleck, N.A.: Ferroelectric switching: a micromechanics model versus measured behaviour. Eur. J. Mech. A. Solids 23, 203-217 (2004)

[8] Li, J., Weng, G.J.: A theory of domain switch for the nonlinear behavior of ferroelectrics. Proc. R. Soc. London A 455, 3493-3511 (1999)

[9] Li, J., Weng, G.J.: A micromechanics-based hysteresis model for ferroelectric ceramics. J. Intel. Mater. Syst. Struct. 12, 79-91 (2001)

[10] Li, W.F., Weng, G.J.: A theory of ferroelectric hysteresis with a superimposed stress. J. Appl. Phys. 91, 3806-3815 (2002)

[11] Li, W.F., Weng, G.J.: A micromechanics-based thermodynamic model for the domain switch in ferroelectric crystals. Acta Mater. 52, 2489-2496 (2004)

[12] Su, Y., Weng, G.J.: The shift of Curie temperature and evolution of ferroelectric domain in ferroelectric crystals. J. Mech. Phys. Solids 53, 2071-2099 (2005)

[13] Su, Y., Weng, G.J.: A self-consistent polycrystal model for the spontaneous polarization of ferroelectric ceramics. Proc. R. Soc. Lond. A 462, 1763-1789 (2006)

[14] Srivastava, N., Weng, G.J.: A dual-phase homogenization theory for the hysteresis and butterfly-shaped behavior of ferroelectric single crystals. Mech. Mater. 38, 945-957 (2006)

[15] Srivastava, N., Weng, G.J.: A theory of double hysteresis for ferroelectric crystals. J. Appl. Phys. 99, 1-11 (2006)

[16] Su, Y., Weng, G.J.: A polycrystal hysteresis model for ferroelectric ceramics. Proc. R. Soc. Lond. A 462, 1573-1592 (2006)

[17] Chan, K., Hagood, N.: Modeling of nonlinear piezoceramics for structural actuation. In: Proceedings of SPIE Symposium on Smart Structures and Materials, vol. 2190, pp. 194-205 (1994)

[18] Steinkopff, T.: Finite-element modeling of ferroelectric domain switching in piezoelectric ceramics. J. Eur. Ceram. Soc. 19, 1247-1249 (1999)

[19] Hwang, S.C., Huber, J.E., McMeeking, R.M., Fleck, N.A.: The simulation of switching in polycrystalline ferroelectric ceramics. J. Appl. Phys. 84, 1530-1540 (1998)

[20] Zhang, Z.K., Fang, D.N., Soh, A.K.: A new criterion for domain-switching in ferroelectric materials. Mech. Mater. 38, 25-32 (2006)

[21] Shaikh, M.G., Phanish, S., Sivakumar, S.M.: Domain switching criteria for ferroelectrics. Comput. Mater. Sci. 37, 178-186 (2006)

[22] Chen, X., Fang, D.N., Hwang, K.C.: Micromechanics simulation of ferroelectric polarization switching. Acta Mater. 45, 3181-3189 (1997)

[23] Lu, W., Fang, D.N., Hwang, K.C.: Micromechanics of ferroelectric domain switching behavior. Part I: Coupled electromechanical field of domain inclusions. Theor. Appl. Fract. Mech. 37, 29-38 (2001)

[24] Lu, W., Fang, D.N., Hwang, K.C.: Micromechanics of ferroelectric domain switching behavior. Part II: Constitutive relations and hysteresis. Theor. Appl. Fract. Mech. 37, 39-47 (2001)

[25] Huber, J.E.: Micromechanical modeling of ferroelectrics. Curr. Opin. Solid State Mater. Sci. 9, 100-106 (2005)

[26] Chen, P.J., Peercy, P.S.: One dimensional dynamic electromechanical constitutive relations of ferroelectric materials. Acta Mech. 31, 231-241 (1979)

[27] Chen, P.J., Madsen, M.M.: One dimensional polar response of the electrooptic PLZT 7/65/35 due to domain switching. Acta Mech. 41, 255-264 (1981)

[28] Chen, P.J., Mongomery, S.T.: A macroscopic theory for the existence of the hysteresis and butterfly loops in ferroelectricity. Ferroelectrics 23, 199-208 (1980)

[29] Chen, P.J., Tucker, T.J.: Determination of the polar equilibrium properties of the ferroelectric ceramic PZT 65/35. Acta Mech. 38, 209-218 (1981)

[30] Bassiouny, A.F., Ghaleb, G., Maugin, G.: Thermodynamical formulation for coupled electromechanical hysteresis effects - I Basic equations. Int. J. Engng. Sci. 26, 1279-1295 (1988)

[31] Bassiouny, A.F., Ghaleb, G., Maugin, G.: Thermodynamical formulation for coupled electromechanical hysteresis effects - II Poling of ceramics. Int. J. Engng. Sci. 26, 1297-1306 (1988)

[32] Kamlah, M., Tsakmakis, C.: Phenomenological modeling of the non-linear electromechanical coupling in ferroelectrics. Int. J. Solids Struct. 36, 669-695 (1999)

[33] Kamlah, M., Böhle, U., Munz, D.: On a non-linear finite element method for piezoelectric structures made of hysteretic ferroelectric ceramics. Comput. Mater. Sci. 19, 81-86 (2000)

[34] Kamlah, M., Böhle, U.: Finite element analysis of piezoceramics components taking into account ferroelectric hysteresis behavior. Int. J. Solids Struct. 38, 605-633 (2001) 
[35] Kamlah, M., Wang, Z.G.: A thermodynamically and microscopically motivated constitutive model for piezoceramics. Comput. Mater. Sci. 28, 409-418 (2003)

[36] Landis, C.M., McMeeking, R.M.: A phenomenological constitutive law for ferroelastic switching and a resulting asymptotic crack tip solution. J. Intel. Mater. Syst. Struct. 10, 155-163 (1999)

[37] Cocks, A.C.F., McMeeking, R.M.: A phenomenological constitutive law for the behavior of ferroelectric ceramics. Ferroelectrics 228, 219-228 (1999)

[38] McMeeking, R.M., Landis, C.M.: A phenomenological multi-axial constitutive law for switching in polycrystalline ferroelectric ceramics. Int. J. Engng. Sci. 40, 1553-1577 (2002)

[39] Landis, C.M.: Fully coupled, multi-axial, symmetric constitutive laws for polycrystalline ferroelectric ceramics. J. Mech. Phys. Solids 50, 127-152 (2002)

[40] Landis, C.M., McMeeking, R.M.: A self-consistent model for switching in polycrystalline ferroelectrics: electrical polarization only. In: Proceedings of the 1999 SPIE Smart Materials Symposium, vol. 3667, pp. 172-180 (2000)

[41] Huber, J.E., Fleck, N.A., Landis, C.M. et al.: A constitutive model for ferroelectric polycrystals. J. Mech. Phys. Solids 47, 1663-1697 (1999)

[42] Schröder, J., Gross, D.: Invariant formulation of the electro-mechanical enthalpy function of transversely isotropic piezoelectric materials. Arch. Appl. Mech. 73, 533-552 (2004)

[43] Schröder, J., Romanowski, H.: A thermodynamically consistent mesoscopic model for transversely isotropic ferroelectric ceramics in a coordinate-invariant setting. Arch. Appl. Mech. 74, 863-877 (2005)

[44] Elhadrouz, M., Zineb, T., Patoor, E.: Constitutive law for ferroelastic and ferroelectric piezoceramics. J. Intel. Mater. Syst. Struct. 16, 221-263 (2005)

[45] Kim, S.J.: A one-dimensional continuum model for ferroelectric ceramics. Eur. J. Mech. A Solids 22, 423-432 (2003)

[46] Kamlah, M.: Ferroelectric and ferroelastic piezoceramics-modeling of electromechanical hysteresis phenomena. Continuum Mech. Thermodyn. 13, 219-268 (2001)

[47] Landis, C.M.: Non-linear constitutive modeling of ferroelectrics. Curr. Opin. Solid State Mater. Sci. 8, 59-69 (2004)

[48] Gong, X., Suo, Z.: Reliability of ceramic multilayer actuators: a nonlinear finite element simulation. J. Mech. Phys. Solids 44, 751-769 (1996)

[49] Hom, C.L., Shankar, N.: A finite element method for electrostrictive ceramic devices. Int. J. Solids Struct. 33, 1757-1779 (1996)

[50] Hwang, S.C., Waser, R.: Study of electrical and mechanical contribution to switching in ferroelectric/ ferroelastic polycrystals. Acta Mater. 48, 3271-3282 (2000)

[51] Fang, D.N., Soh, A.K., Liu, J.X.: Electromechanical deformation and fracture of piezoelectric/ ferroelectric materials. Acta Mech. Sin. 17, 193-213 (2001)

[52] Liu, B.: Study on the fracture and fatigue of ferroelectric materials (in Chinese). Dissertation for the Doctoral Degree. Tsinghua University, Beijing, pp. 82-97 (2000)

[53] Ghandi, K., Hagwood, N.W.: A hybrid finite element for phase transitions in nonlinear electromechanically coupled material. In: 97 SPIE Proc. vol. 2339, pp. 97-112 (1997)

[54] Landis, C.M.: A new finite element formulation for electromechanical boundary value problems. Int. J. Numer. Methods Engng. 55, 613-628 (2002)

[55] Li, F.X., Fang, D.N.: Simulations of domain switching in ferroelectrics by a three-dimensional finite element model. Mech. Mater. 36, 959-973 (2004)

[56] Kim, S.J., Jiang, Q.: A finite element model for rate-dependent behavior of ferroelectric ceramics. Int. J. Solids Struct. 39, 1015-1030 (2002)

[57] Arockiarajan, A., Delibas, B., Menzel, A., Seemann, W.: Studies on rate-dependent switching effects of piezoelectric materials using a finite element model. Comput. Mater. Sci. 37, 306-317 (2006)

[58] Kessler, H., Balke, H.: On the local and average energy release in polarization switching phenomena. J. Mech. Phys. Solids 49, 953-978 (2001)

[59] Lynch, C.S.: The effect of uniaxial stress on the electro-mechanical response of 8/65/35 PLZT. Acta Mater. 44, 4137-4148 (1996)

[60] Liu, F., Li, H.J., Wang, T.C.: Energy principle of ferroelectric ceramics and single domain mechanical model. Acta Mech. Sin. 23, 531-543 (2007) 